\title{
Random packing of hyperspheres and Marsaglia's Parking Lot Test
}

\author{
Stefan C. Agapie and Paula A. Whitlock* \\ Department of Computer and Information Sciences \\ Brooklyn College \\ 2900 Bedford Avenue \\ Brooklyn, New York 10021
}

September 30, 2009

\begin{abstract}
Many studies of randomly packed hyperspheres in multiple dimensions have been performed using Monte Carlo or Molecular Dynamics simulations to probe the behaviour of the systems. The calculations are usually initiated by randomly placing the hyperspheres in some D dimensional box until some randomly loosely packed density is achieved. Then either a compression algorithm or a particle scaling technique is used to reach higher packing fractions. The interesting aspect in the initial random placing of the hyperspheres is that it is closely related to a test of random number generators that was proposed by Marsaglia, the "Parking Lot" test. It is this relationship that is investigated in this paper.
\end{abstract}

*whitlock@brooklyn.cuny.edu 


\section{Introduction}

The study of hard hyperspheres in a variety of dimensions has been an active field for many years in part because in two and three dimensions hyperspheres can represent common materials like simple fluids, or non-interacting granular substances such as gravel, beads or fruit. In these systems, most of the structure is determined by the purely repulsive hard sphere interaction. When extended to dimensions greater than three, hard hyperspheres are used to investigate properties such as geometrical frustration and the geometry of the crystalline state. One very interesting property is the likelihood of randomly placed hyperspheres forming jammed states rather than reorganizing into a crystal. Decades of simulation studies using both Monte Carlo (MC) and Molecular Dynamics (MD) have shown that while two dimensional hard disks readily reorganise into a hexagonally ordered state as the density is increased from a fluid density, this is not true for higher dimensions. Instead, increasing the density of a hard hypersphere fluid system for dimensions greater than two results in a jammed state, 1i.e., a state in which the density can not be increased. Various suggestions have been made as to why the transition from a fluid state to an ordered, crystalline state is difficult $[1,2,3,4]$.

To begin a simulation of a hard hypersphere system, an initial configuration of non-overlapping hyperspheres can be obtained by either starting from a crystal state at the chosen density or by randomly placing the hyperspheres in the simulation box. The latter method will only work for low to moderate fluid densities before it becomes too time consuming to find sufficient space where a hypersphere can be placed and not overlap its neighbors. This difficulty has given rise to the development of techniques for achieving higher densities from lower density initial configurations. For example, in their studies of random packing of spheres, Jodrey and Tory[5] used a compression algorithm in which 
the spheres were pressed together, but not allowed to overlap. A similar techniques was used by Speedy [6] to examine the glass transition in three dimensions, and by Lue, Bishop and Whitlock[7] to study four and five dimensional hypersphere systems. Another method involves a particle scaling algorithm[8] in which the hyperspheres are allowed to increase in size until they touch. Then each hypersphere is moved for a set number of passes (MC) or time steps (MD) to re-equilibrate and loose memory of the scaling. Further rescaling is possible until the maximum jamming density is attained.

Our interest in this paper is in the initial random placement of the hyperspheres in their simulation box. This is a system also studied by Marsaglia in the development of his "Parking Lot" test[9] to determine how well a sequence of pseudorandom numbers (prns) from a deterministic generator mimics the properties of a truly random sequence. The Parking Lot test is distributed as part of the Diehard suite of tests of pseudorandom number generators (prng). The suite is freely available on-line[10] and is used by many researchers to decide whether a specific pseudorandom number generator will be adequate for their simulations. In this article, we will use Marsaglia's original version of the "Parking Lot" test, our rewriting of the algorithm and our extension of it to multiple dimensions. We investigate the properties of the various versions of the "Parking Lot" test with three different types of pseudorandom number generators. We will discuss why Marsaglia choose to analyze the results as he did and relate this to the difficulty of achieving high densities of hypersphere placement by random "parking" alone. Finally, we will discuss whether the choice of pseudorandom number generator for creating the initial configuration for a simulation will likely affect the final results. 


\section{Parking Lot Tests and Random Packing}

\subsection{Marsaglia's Parking Lot Test}

Marsaglia developed the Parking Lot and related tests to determine the uniformity of placement of hyperspheres in D dimensional space when the coordinates of the center of the hyperspheres are the result of successive calls to a prng. The "parking problem" is a well-known random packing problem and was given an exact mathematical treatment in one dimension by Rényi[11]. However, the generalization to more than one dimension is less tractable. Marsaglia's test is a straightforward placing of a hyperspherical or hypercubic "car" or particle of a predetermined size in a D-dimensional box without overlapping any of the previously parked particles. Let $\overrightarrow{c_{1}}, \overrightarrow{c_{2}}, \ldots, \overrightarrow{c_{k}}$ be the coordinates of the $k$ previously non-overlapping parked particles. Then the coordinates of a next possible particle are generated. The position of the new particle is compared with the $k$ other particles to check for overlap. If overlap occurs, the particle is rejected, otherwise it becomes the $k+1$ successfully parked particle. After $n$ attempts, there will be $k$ successfully placed particles. Marsaglia[9] was not able to determine the expected distribution of $k$ given $n$, but was able to show that plots of $k$ vs $n$ gave very different curves for different prngs.

In the Diehard test suite, Marsaglia chose to perform the Parking Lot test in two dimensions for a specific size box with a specific value for $n$. Using a square box of side $L=100, n=12000$ particles are attempted to be placed in the box. As long as the newly proposed particle does not overlap any previously placed particle and its center is inside the simulation box, the new particle is accepted. Marsaglia had previously performed a simulation with what he considered to be a very good prng and had found that $k$ should have an average value of 3523 with a standard error of 21.9. He asserted in both the Diehard test suite[10] and a later publication[12] that the distribution of $(k-3523) / 21.9$ should be 
normal. In both references, the particle is described as a disk of radius 1.0. However, the Diehard Fortran code reveals that a square of side 1.0 was used:

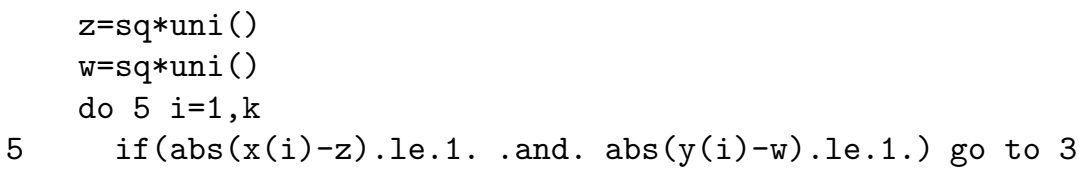

The line labelled 5 is a test for the overlap of two squares. If disks were being used, the distance between the particles $r=\sqrt{(x(i)-z)^{2}+(y(i)-z)^{2}}$ would need to be less than or equal to one. Does it matter whether one uses disks or squares? An indication of the importance of which geometric figure is parked can be obtained by comparing the area occupied by a square of side 1.0 to the area of a disk of diameter 1.0. The ratio of the areas, disk to square, is $\pi / 4$. Therefore, it would be anticipated that more disks could be placed in a box than squares in the same number of tries. Marsaglia may have chosen to use squares for the Parking Lot test because of the previous work on the packing density of $n$-dimensional unit hypercubes[13, 14, 15] and his own work on the distribution of distances between random points in rectangles[16].

\subsection{Extensions of the Parking Lot Test}

In the research reported here, Marsaglia's original Parking Lot test code was translated from Fortran to $\mathrm{C}++$. This involved some interpretation since the Diehard Test suite preprocesses the output from a prng so that all the tests use the same bitstream file as input. That is, the user of the Diehard Test suite needs to first create a file of 32-bit integers obtained from the prng to be tested. These are written out in a hexadecimal format. Another code translates the hexadecimal numbers into a binary file which is then used as input for the tests. Preprocessing was also done for the $\mathrm{C}++$ code. Each prng was called according 
to its specifications to return a number between 0 and 1 . These floating point values were translated into 32 or 48 bit integers ${ }^{1}$ and written to an output file in binary. The binary file was used as input for all the $\mathrm{C}++$ versions of the parking lot test. As a further test, a $\mathrm{C}$ code was written that did not do such preprocessing of the output from a prng. Rather, it used the values between 0 and 1 provided by the prng to place squares in the simulation box.

Employing the same preprocessed file as in the parking squares test, a parking of disks was coded. Using the same specifications as in the original test, a simulation box with sides $L=100$ and $n=12000$ attempts, disks with diameter 1.0 were placed. A prng was used to obtain the random coordinates of the center of mass of a new disk to be put in the box. If it did not overlap any previously placed disk and if its center was within the simulation box, the new disk was accepted. Otherwise, it was rejected. As anticipated, the number of disks that could be placed was larger than the number of squares in the same size simulation box. The fraction of space occupied by the disks is significantly less than that of a completely packed or jammed state. Of course, through a rejection process alone, the jammed state can not be reached[17].

The generalization to three and four dimensions was straightforward. In each case, hyperspheres for the chosen dimension were placed in simulation boxes such that no overlap between hyperspheres was allowed. The size of the simulation boxes and the number of attempts to place hyperspheres needed to be specified. It was decided to keep similar volumes in the multidimensional spaces and attempt to place the same number of hyperspheres as in the two dimensional case, $n=12000$. In three dimensions, the size of the cubic simulation box was chosen to be $L=22.0$ which yields a volume of 10648.0. In four dimensions, the hypercubic box had a side of $L=10.0$. As the dimension increased with similar

\footnotetext{
${ }^{1}$ All calculations were performed on a 64 bit processor where either 32 bit or IEEE arithmetic could be chosen
} 
internal hypervolumes for the simulation box, the number of hyperspheres that could be placed in 12,000 tries decreased.

\section{Pseudorandom Number Generators}

In investigating and extending the Parking Lot test three pseudorandom number generators were utilized. The first was a multiplicative congruential generator, called rannyu, that was developed in the 1960's to run on the CDC 6600 which had 48 bit integers:

$$
X_{n+1}=M X_{n} \quad \bmod 2^{48}
$$

where $M$ was chosen to have good spectral properties[19]. Since this generator does not have an additive constant, its period (length of the sequence before numbers begin to repeat) is less than the maximum of $2^{48}-1$. It has been extensively tested by empirical tests and its shortcomings are well known[20]. For example, upon applying the Diehard tests, rannyu fails the Overlapping-PairsSparse-Occupancy, the Overlapping-Quadruples-Sparse-Occupancy and the DNA tests for bits 20-32 of a 32 bit word. A more thorough description is given in Schmidt[18]. An optimized code for the prng was originally written in Fortran, but has since been translated into $\mathrm{C}, \mathrm{C}++$ and Java. A $\mathrm{C}++$ version of the code was used here.

The second generator was of a completely different type and is based on the Weyl sequence[21]

$$
X_{n}=\{n \alpha\}
$$

where the curly brackets \{\} indicate the fractional part of the irrational number $\alpha$ and $n=1,2, \ldots$. Weyl showed that the numbers produced by this sequence were uniform on $(0,1)$. However, it has serious correlations and a generalization 
called the "Shuffled Nested Weyl Sequence" (SNWS)[22], given by

$$
\begin{aligned}
& \nu_{n}=M\{n\{n \alpha\}\}+0.5, \\
& Z_{n}=\left\{\nu_{n}\left\{\nu_{n} \alpha\right\}\right\} .
\end{aligned}
$$

is used as a pseudorandom number generator instead. The multipler $M$ is a large integer and again $\alpha$ is an irrational number. The SNWS passes all the Diehard tests. In the paper where the SNWS generator was introduced, Holian et al[22] mention that a poor choice of prng used to initialize a MD trajectory can lead to an unphysical result despite the Lyapunov instability[23] criteria. The Lyapunov instability suggests that the initial state is forgotten after a few collision times.

The third prng is the $2003 \mathrm{C}++$ version of the well-regarded "Mersenne Twister" (MT) generator first published in 1998 by Matsumoto and Nishimura[24]. The MT generator is a multiple-recursive matrix method[25, 26]. A random vector sequence is generated over the two element field $F_{2}=\{0,1\}$ by the linear recurrence,

$$
\vec{x}_{k+n}=\vec{x}_{k+m}+\vec{x}_{k+1}\left(\begin{array}{cc}
0 & 0 \\
0 & I_{r}
\end{array}\right) A+\vec{x}_{k}\left(\begin{array}{cc}
I_{w-r} & 0 \\
0 & 0
\end{array}\right) A .
$$

The integer $n$ is the degree of the recurrence, the integer $m$ is $1 \leq m \leq n, w$ is the machine word size, and $0 \leq r \leq w-1$. The $I_{r}$ and $I_{w-r}$ are idenity matrices of size $r$ and $w-r$, respectively, and $A$ is a $w \times w$ matrix with elements on $F_{2}$. Each vector is multiplied by a $w \times w$ invertible matrix $T$ on the right in a process called tempering[27]. This is done to improve the $k$-distribution properties of the least significant bits of the vector. The specific version of the generator is 623 -distributed to 32 bits accuracy and has a period of $2^{19937}-1$. 
Matsumoto and Nishimura report that the MT generator passed the Diehard suite of tests.

\section{Results}

The Diehard testing suite version of the parking test was applied to each of the prngs discussed above. The results are shown in the first line of Table I. All three generators give values for the mean and sigma in agreement well within their standard errors with Marsaglia's values of $\mu=3523$ and $\sigma=21.9$. The $\mathrm{C}++$ code's results, the second line in Table I, are similar but not identical to the Diehard test suite results for several reasons. First, the preprocessing is not identical and the initial state of the generator is not the same for the two sets of tests. Futhermore, the $\mathrm{C}++$ code computes an average of 1000 repetions of a single parking test while the Diehard testing suite repeats the test 10 times. Of course, the values of $\mu$ and $\sigma$ should not be dependent on the preprocessing or the initial state of the generator since these are the parameters of the normal distribution representing the asymptotic distribution function for the process, i.e. trying to place 12,000 squares in a box of side $L=100$. So, it is no surprise that the $\mathrm{C}$ code which uses no preprocessing gives a value almost the same as that obtained by the $\mathrm{C}++\operatorname{code}^{2}$.

The fourth line of Table I lists the outcome when disks rather than squares are placed in the simulation box. As expected the number of disks placed, $\mu \approx 4088$, is greater than the number of squares placed, $\mu \approx 3523$. The observed ratio being $\sim 0.86$ which is somewhat larger than $\pi / 4$. In his initial description of the parking lot test[9], Marsaglia found that shift-register pseudorandom number generators gave very different behaviors than combination prngs when

\footnotetext{
${ }^{2}$ The values are fortuitously the same. An independent run of the code for another 1000 repetitions of the test gave $\mu=3525.1$ and $\sigma=21.7$
} 
$k$ was plotted as a function of $n$. In Figure 1, the behavior of $k(n)$ for the three prngs considered here are plotted. On the scale displayed, the prngs all seem to show identical behavior. Some minor differences are observed for larger values of $n$ as shown in Figure 2; the SNWS generator successfully places fewer disks for large $n$. Calculating values for $\mu$ and $\sigma$ implies that the asymptotic distribution for placing the disks is a normal distribution. This assertion is examined in Figure 3 where the frequency of the value of $k$ observed in 1000 repetitions of the parking disks test is graphed. For comparison, a normal distribution using the averaged value of $k$ as $\mu$ and its standard error as $\sigma$ for the Mersenne Twister prng is shown.

In Table II, as anticipated, the average number of placed hyperspheres in three and four dimensions is less than the average in two dimensions. Though the hypervolumes are numerically similar in all the dimensions considered, the reduced length of a side of the simulation box as the dimensions increases, restricts the open space in which to randomly place the hyperspheres. The affect of the side length on placing hyperspheres in three and four dimensions can be ascertained by changing the side of the simulation box in both dimensions to $\mathrm{L}$ $=100.0$. This change corresponds to reducing the number density to very low values, $\rho=0.012$ in three $\mathrm{D}$ and $\rho=0.00012$ in four D. In both cases 12,000 hyperspheres were easily placed. Another interesting aspect of the results in Table II is the similarity of the $\sigma$ values for all three prngs. This behavior is reinforced by viewing the variation of $k$ with $n$ shown in Figure 4 for three D and in Figure 7 in four-D. Except for the largest values of $n, k(n)$ for the three prngs is almost identical. When the range, $11500 \leq n \leq 12000$ us examined, the SNWS consistently places less hyperspheres thant the MT prng, see Figure 5 and Figure 8. The significance of this small but consistent behavior is not clear since all values in the graphs are within each other's standard error. 
The issue of the distribution of the observed values of $k$ is addressed in Figures 6 and 9. Figure 6 graphs the frequencies of 1000 observed values of $k$ when $n=12,000$ and compares them with a normal distribution, $\phi(k) \propto \exp ((k-$ $\left.3857.1)^{2} /(23.1)^{2}\right)$ for the SNWS prng in three dimensions. In four dimensions, the frequency function comparisons of observed $k$ values after 12, 000 tries obtained from the MT prng is compared with $\phi(k) \propto \exp \left((k-3724.8)^{2} /(25.6)^{2}\right)$. The agreement is very good and helps to convince that Marsaglia's assertion that the distribution of the average value of $k$ in two dimensions is a normal distribution.

\section{Discussion}

Why did Marsaglia choose the specific settings that he did for the parking lot test? Why not choose to test for the well-researched limiting packing fraction for a two-dimensional system? The latter is an elusive value that is dependent on the speed of the processor, the memory available and the time that a researcher is willing to wait. In 1979, it was claimed that only packing fractions below 0.5473 could be achieved by purely random placement of disks[17]. Thirty years later, on very much faster processors and a willingness to wait hours to achieve a particular value, still leads to a packing fraction of only 0.5498. After $k$ disks have been added to the simulation box, the residual space available to the next disk is $R_{k}$. The probability that a proposed next disk finds sufficient space without overlapping is proportional to the area of $R_{k}$ and this deceases steadily with the increasing value of $k$. This is a geometric restriction and as $k$ increases, the number of tries needed to place the next disk rises at a rate more than expoenential. So, the limiting randomly achieved packing fraction, while well-studied is not a good candidate for a fast, easily implemented test of pseudorandom numbers. 
The implementation

\section{Acknowledgements}

We thank Professor Marvin Bishop for helpful discussions. One of us, S.C.A., was partially supported by PSC/CUNY Award 61519-00 39.

\section{References}

[1] E.I. Corwin, H.M. Jaeger, and S.R. Nagel, "Structural signature of jamming in granular media," Nature, $\underline{435}, 1075-1078,(2005)$.

[2] M. Skoge, A. Donev, F.H. Stillinger, and S. Torquato, "Packing hyperspheres in high-dimensional Euclidean spaces," Phys. Rev. E, 푸, 041127-11 (2006).

[3] C. Song, P. Wang, and H.A. Makse, "A phase diagram for jammed matter," Nature, $\underline{453}, 629-632,(2008)$.

[4] J.A. van Meel, D. Frenkel, and P. Charbonneau, "Geometrical Frustration: A Study of 4d Hard Spheres," ? (2009).

[5] W.S. Jodrey and E.M. Tory, "Computer simulation of close random packing of equal spheres," Phys. Rev. A, $\underline{32}, 2347$ (1985).

[6] R.J. Speedy, "On the reproducibility of glasses," J. Chem. Phys., 100, 6684 (1994).

[7] L. Lue, M. Bishop and P.A. Whitlock, "The Fluid to Solid Phase Transition of Hard Hyperspheres in Four and Five Dimensions," submitted to J. Chem. Phys., (2009).

[8] D.P. Fraser, "Setting up Random Configurations," Information Quarterly for Computer Simulation of Condensed Phases," 19, 53-59 (1985).

[9] G. Marsaglia, "A Current View of Random Number Generators," In Computer Science and Statistics: Proceedings of the Symposium on the Interface, 16th, Atlanta, Georgia, March 1984 (Ed. L. Billard). New York: Elsevier, 1985.

[10] G. Marsaglia," The Marsaglia Random Number CDROM, with the Diehard Battery of Tests of Randomness, http://www.stat.fsu.edu/pub/diehard/. 
[11] A. Rényi, "On a one-dimensional problem concerning random space filling," Publ. Math. Inst. Hung. Acad. Sci. 모 109-127 (1958).

[12] G. Marsaglia, "On the randomness of Pi and other decimal expansions," Interstat, October 2005, \#5, http://interstat.statjournals.net/YEAR/2005/articles/0510005.pdf

[13] H. Solomon, "Random packing density," Proc. Fifth Berkeley Symp. Math. Statist. and Prob., $\underline{3}, 119-134$ (1967).

[14] B.E. Blaisdell and H. Solomon, "On random sequential packing in the plane and a conjecture of Palásti," J. of Appl. Prob., $\underline{7}, 667-689,(1970)$.

[15] Y. Akeda and M. Hori, "Numerical test of Palásti's conjecture on twodimensional random packing density," Nature, 254, 318-319 (1975).

[16] G. Marsaglia, B. Narasimhan and A. Zaman, "The distance between random points in rectangles," Comm. Stat.-Theory Meth., 19, 4199-4212 (1990).

[17] M. Tanemura, "On random complete packing by discs," Ann. Inst. Stat.

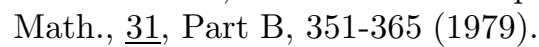

[18] K.E. Schmidt, "Variational and green's function Monte Carlo calculations of few-body systems," in Models and Methods in Few-Body Physics, Lecture Notes in Physics, 273, 363-407 (1987).

[19] R. R. Coveyou and R. D. Macpherson, "Fourier Analysis of Uniform Random Number Generators," J. of the ACM, 14, 100-119, (1967).

[20] O.E. Percus and J. Percus, "Long range correlations in linear congruential generators," J. of Comp. Phys., 그, 267-269 (1988).

[21] H. Weyl, "Ueber die Gleichverteilung von Zahlen mod Eins," Math. Ann. 77, 313-352 (1916).

[22] B.L. Holian, O.E. Percus, T.T. Warnock and P.A. Whitlock, "Pseudorandom number generator for massively parallel molecular-dynamics simulations," Phys. Rev. E 무, 1607-1615 (1994).

[23] B.L. Holian, W.G. Hoover and H.A. Posch, "Resolution of Loschmidt's paradox: The origin of irreversible behavior in reversible atomistic dynamics," Phys. Rev. Lett. $\underline{59}, 10-13$ (1987).

[24] M. Matsumoto and T. Nishimura, "Mersenne Twister: A 623Dimensionally Equidistributed Uniform Pseudo-Random Number Generator," ACM Transactions on Modeling and Computer Simulation, $\underline{8}$ 3-30 (1998).

[25] H. Niederreiter, "Factorization of polynomials and some linear-algebra problems over finite fields," Linear Algebra Appl., 192, 301-328 (1993). 
[26] H. Niederreiter, "The multiple-recursive matrix method for pseudorandom number generation," Finite Fields Appl., 1, 3-30 (1995).

[27] M. Matsumoto and Y. Kurita, "Twisted GFSR generators II," ACM Trans. Model. Comput. Simul., $\underline{4}$, 254-266 (1994). 
Table I Two-Dimensional Parking Problem, average number parked

\begin{tabular}{lccc}
\hline \hline Specific Test & rannyu & SNWS & MT \\
\hline Diehard Suite (squares) & $3533.7 \pm 25.1$ & $3529.0 \pm 23.2$ & - \\
C++ code parking squares & $3523.9 \pm 21.9$ & $3522.6 \pm 22.6$ & $3524.2 \pm 21.7$ \\
C code parking squares & $3523.6 \pm 21.9$ & - & - \\
C++ code parking disks & $4088.3 \pm 25.6$ & $4086.3 \pm 24.1$ & $4087.6 \pm 23.5$ \\
\hline \hline
\end{tabular}

Table II Average number of hyperspheres placed in three and four dimensions.

\begin{tabular}{lccc}
\hline \hline $\begin{array}{l}\text { Number of } \\
\text { dimensions }\end{array}$ & rannyu & SNWS & MT \\
\hline 3 & $3857.3 \pm 24.3$ & $3857.1 \pm 23.1$ & $3858.5 \pm 23.0$ \\
4 & $3724.3 \pm 24.3$ & $3724.0 \pm 25.8$ & $3724.8 \pm 25.6$ \\
\hline \hline
\end{tabular}




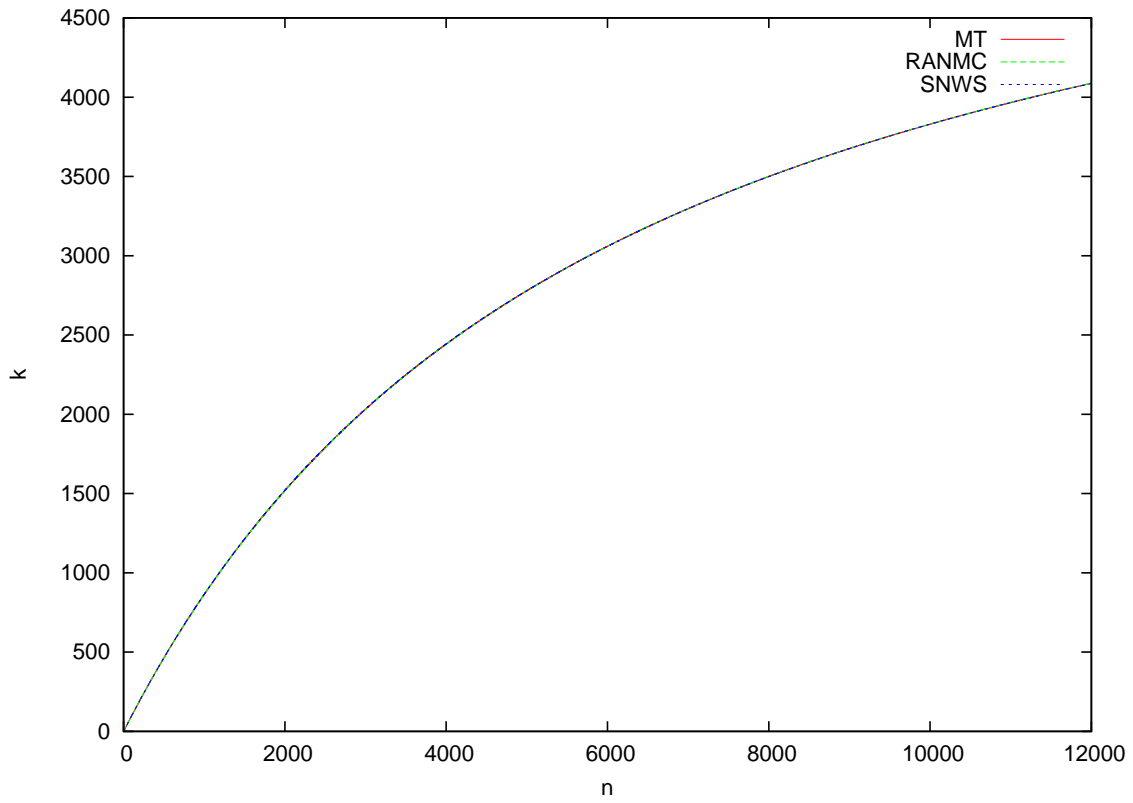

Figure 1: Comparing the number of successfully placed disks, $k$, versus the number of tries, $n$.

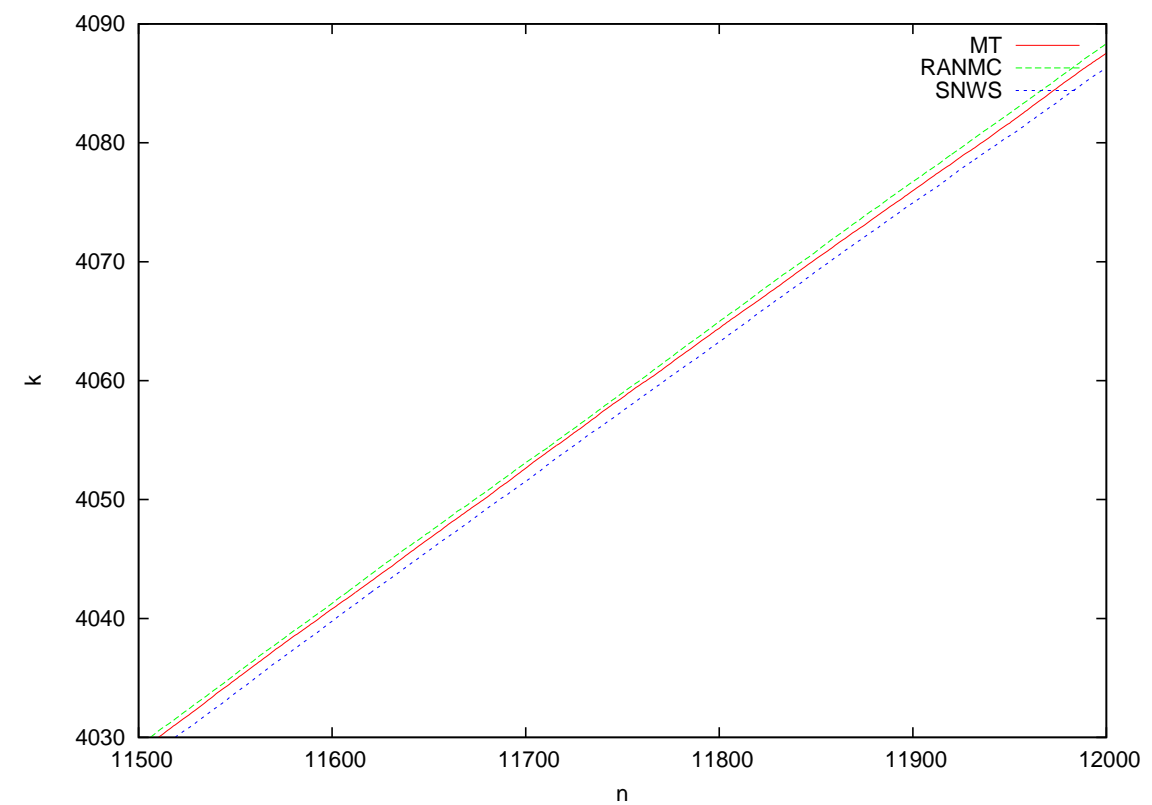

Figure 2: Comparing the number of successfully placed disks, $k$, versus the number of tries for large values of $n$ 


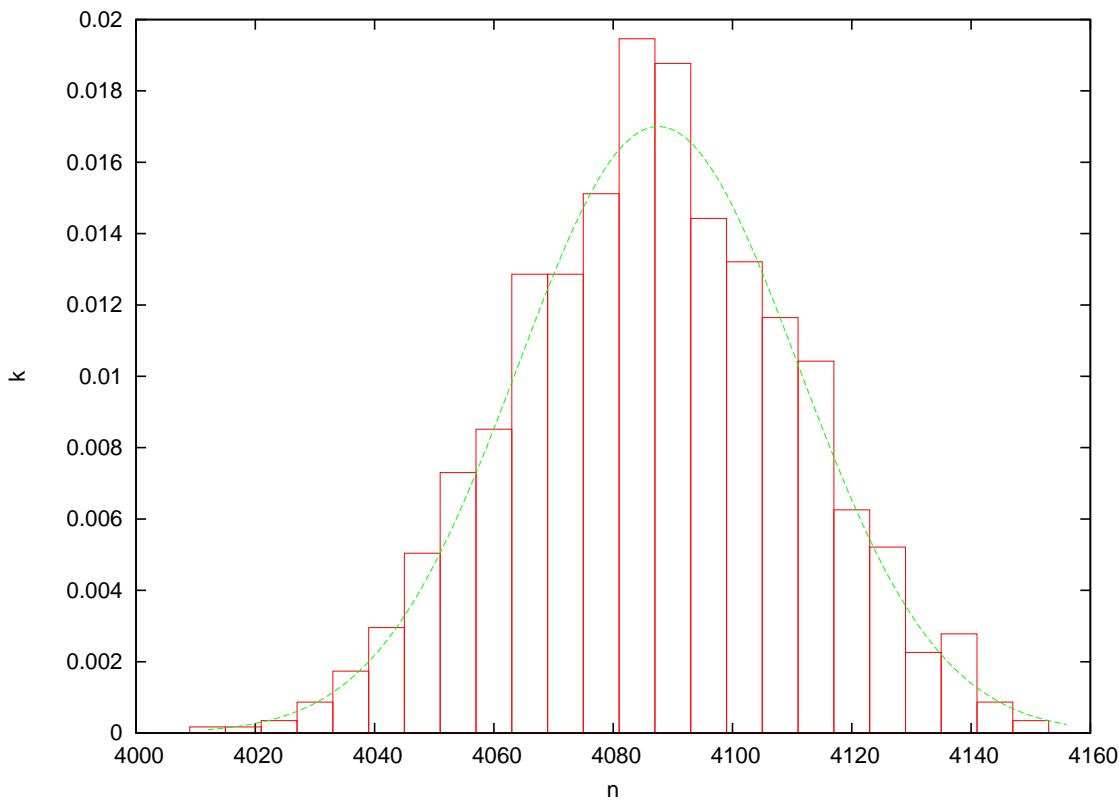

Figure 3: Frequency of values of $k$ for placing disks in a simulation box with the Mersenne Twister prng. The dashed line is a normal distribution using $\mu=4087.6$ and $\sigma=23.5$ 


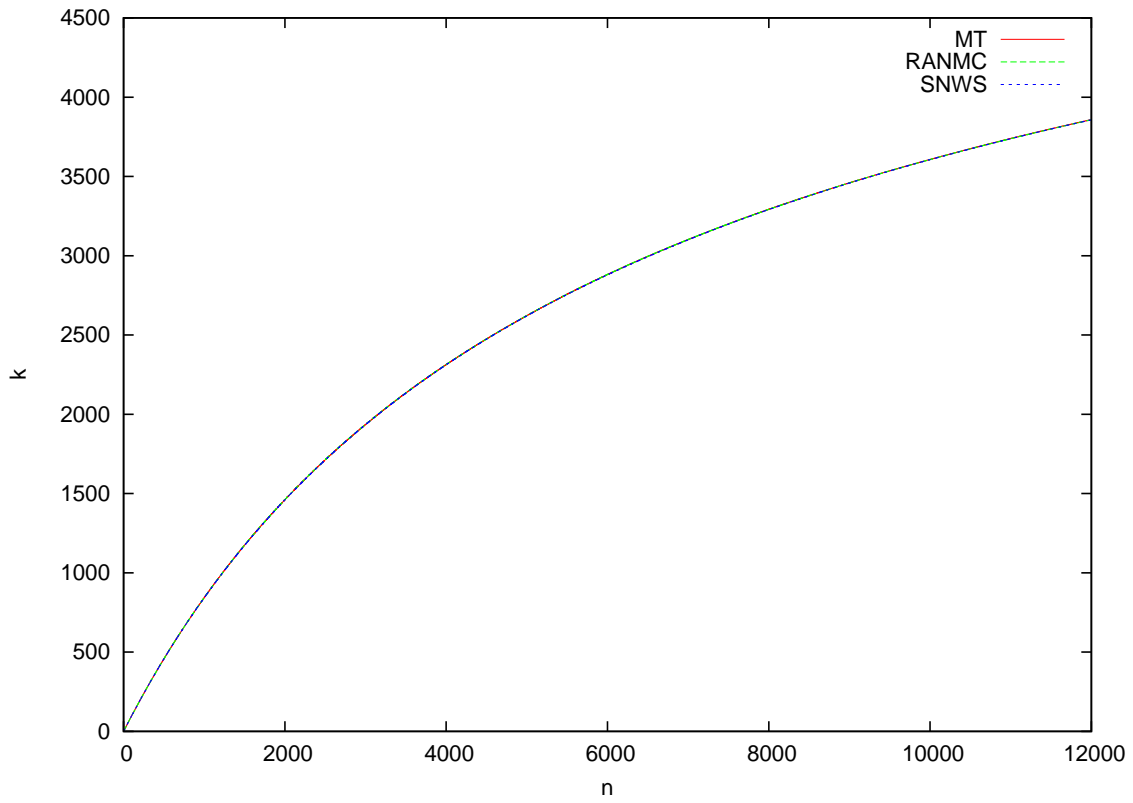

Figure 4: Comparing the number of successfully placed spheres, $k$, versus the number of tries, $n$.

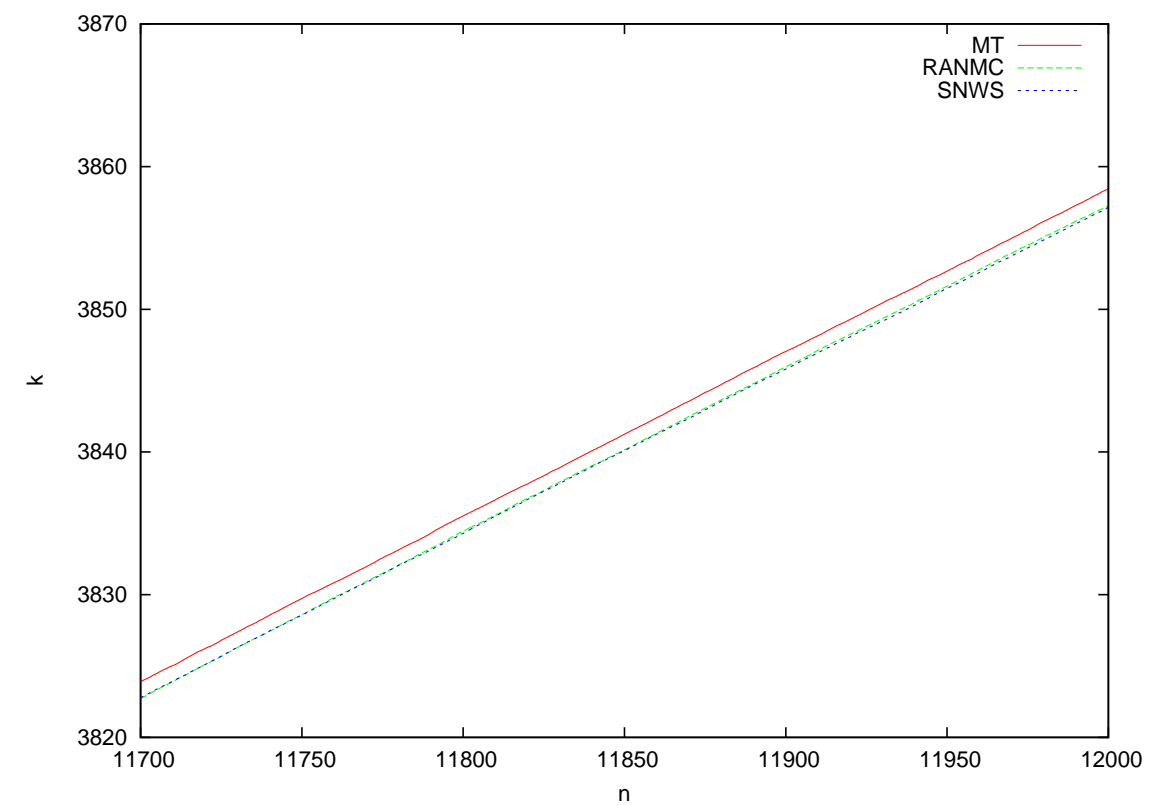

Figure 5: Comparing the number of successfully placed spheres, $k$, versus the number of tries for large values of $n$ 


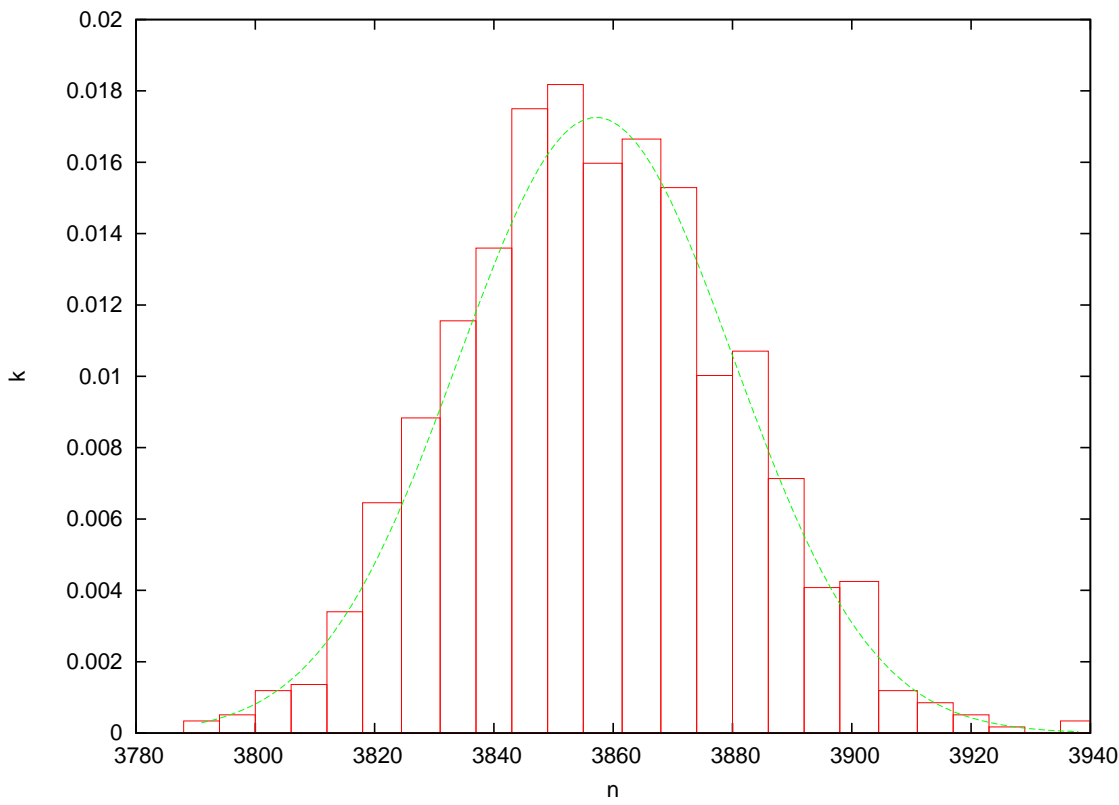

Figure 6: Frequency of values of $k$ for placing spheres in a simulation box with the Shifted Nested Weyl prng. The dashed line is a normal distribution using $\mu=3857.1$ and $\sigma=23.1$ 


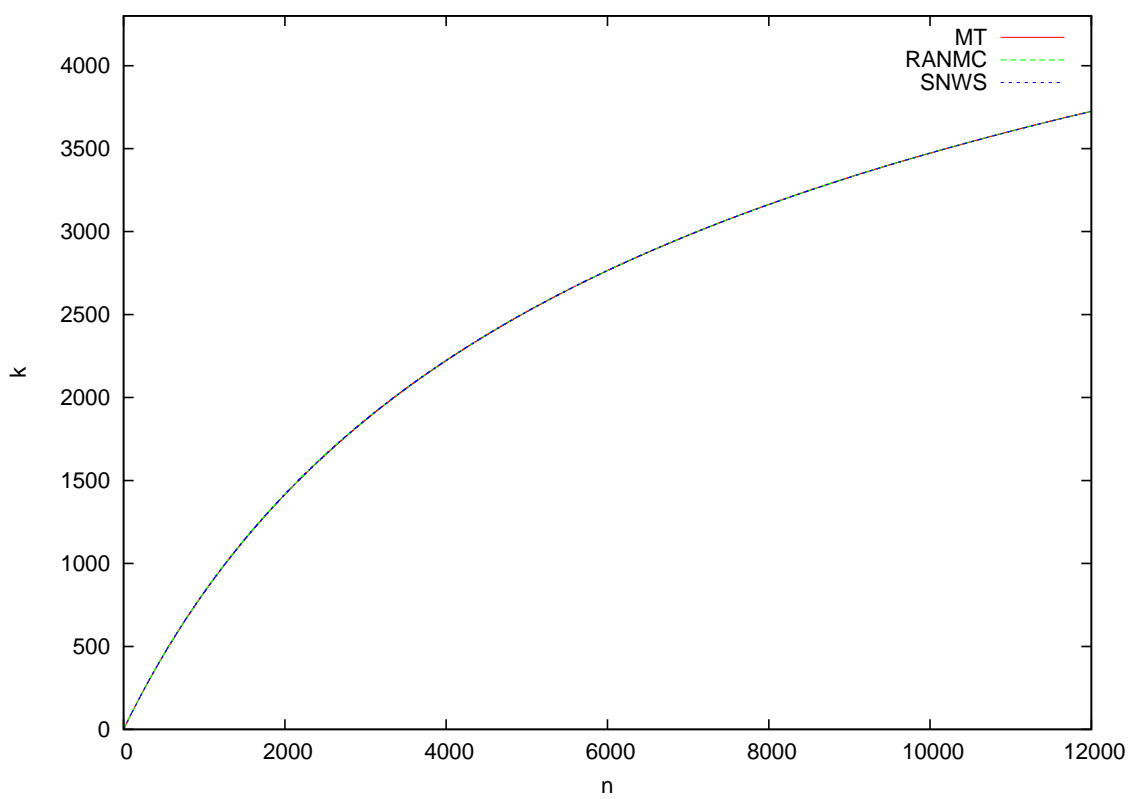

Figure 7: Comparing the number of successfully placed 4-D hyerspheres, $k$, versus the number of tries, $n$.

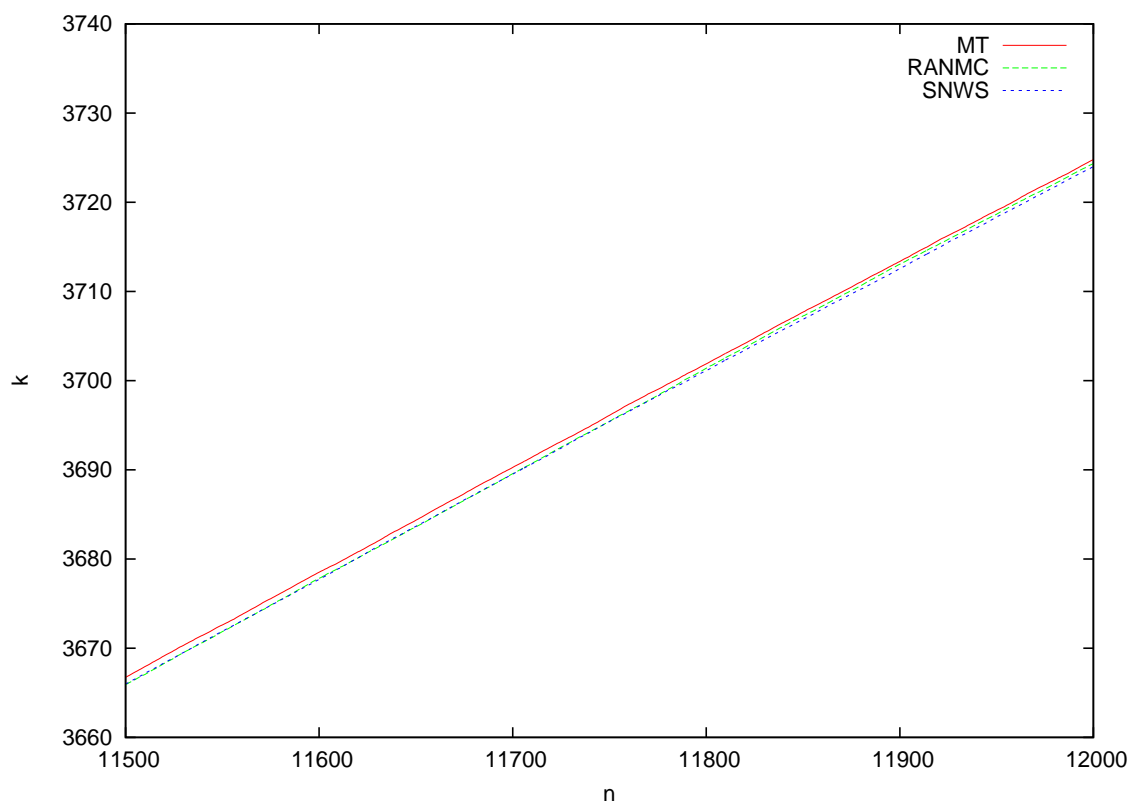

Figure 8: Comparing the number of successfully placed 4-D hyperspheres, $k$, versus the number of tries for large values of $n$ 


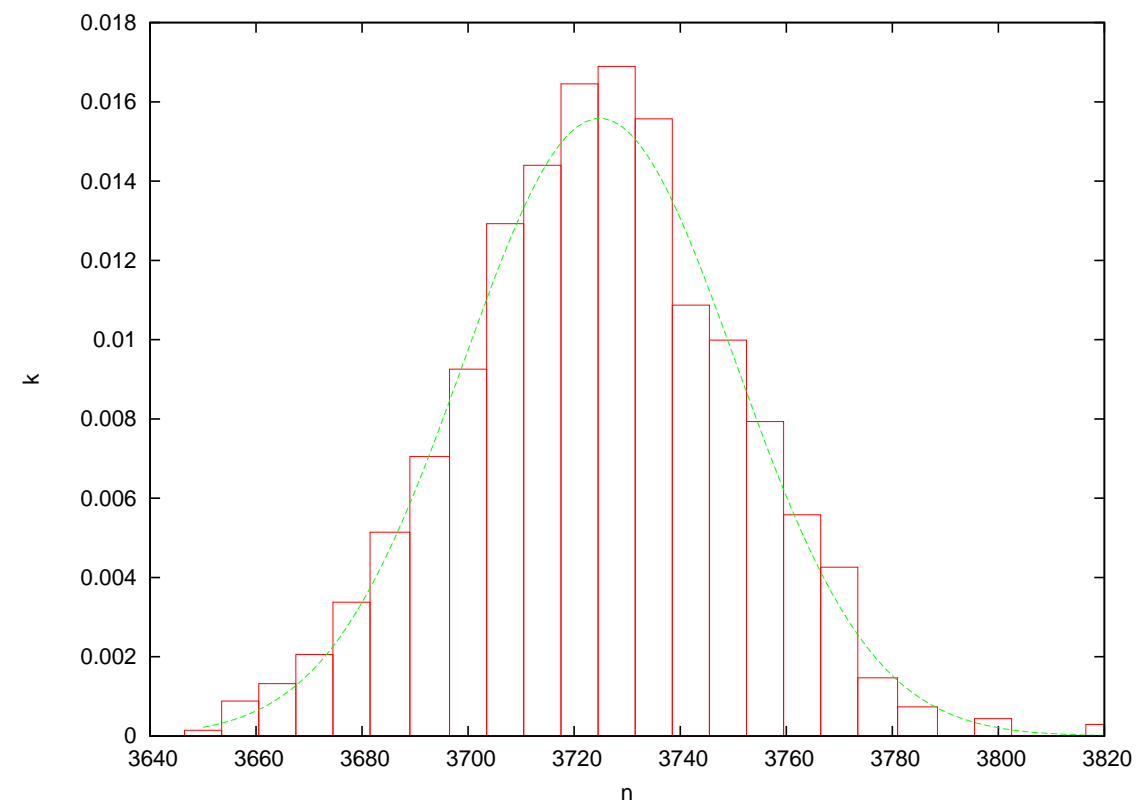

Figure 9: Frequency of values of $k$ for placing 4-D hyperspheres in a simulation box with the Mersenne Twister prng. The dashed line is a normal distribution using $\mu=3724.8$ and $\sigma=25.6$ 